\title{
Computer simulation of wetting, capillary forces, and particle- stabilized emulsions: From molecular-scale to mesoscale modeling
}

\author{
$\operatorname{AUTHOR}(\mathrm{S})$ : \\ Shinto, Hiroyuki
}

\section{CITATION:}

Shinto, Hiroyuki. Computer simulation of wetting, capillary forces, and particle-stabilized emulsions: From molecular-scale to mesoscale modeling. Advanced Powder Technology 2012, 23(5): 538-547

ISSUE DATE:

2012-09

URL:

http://hdl.handle.net/2433/160279

\section{RIGHT:}

(C) 2012 The Society of Powder Technology Japan. Published by Elsevier B.V.; This is not the published version. Please cite only the published version.; この論文は出版社版でありません。引用の際には出版社版を ご確認ご利用ください。 


\title{
Computer simulation of wetting, capillary forces, and
}

\section{particle-stabilized emulsions: from molecular-scale to}

\section{mesoscale modeling}

\author{
Hiroyuki Shinto*
}

Department of Chemical Engineering, Kyoto University, Katsura, Nishikyo-ku, Kyoto 615-8510, Japan

Phone: +81-75-383-2671, Fax: +81-75-383-2651, e-mail: shinto@ cheme.kyoto-u.ac.jp 


\begin{abstract}
The present paper gives an overview of two methodologies for the computer simulation of wetting and capillary forces as well as particle-stabilized emulsions, which usually appear in a two-phase fluid system including solid bodies. One method is the molecular simulation with a coarse-grained model. The other is the computational fluid dynamics with a phase-field model. The main emphasis of this review is concerning our idea how to model the solid surfaces with wettability for the different simulation methods in the same spirit of "a simple modeling with focusing on hydrophilicity and hydrophobicity".
\end{abstract}

Keywords:

Wettability; Capillary forces; Molecular simulations; Coarse-grained models Computational fluid dynamics; Phase-field model. 
Graphical Abstract

Molecular-scale modeling

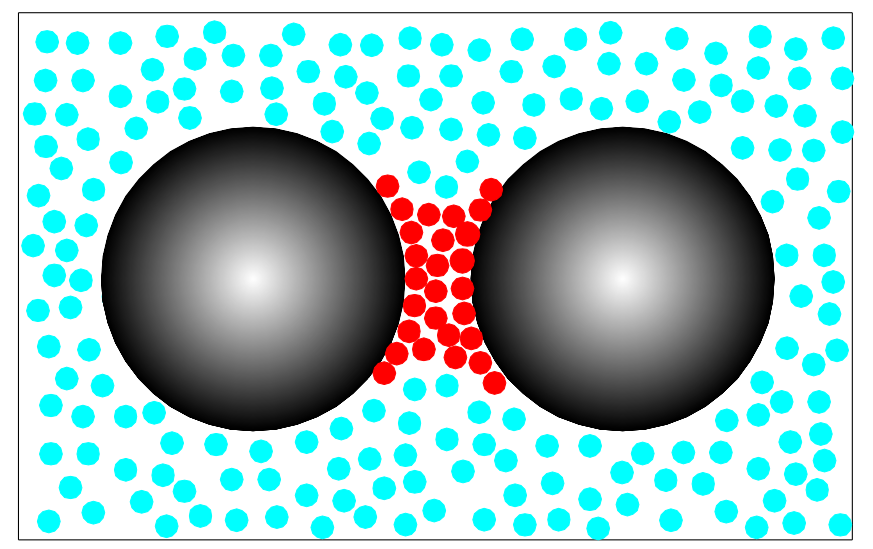

Mesoscale modeling

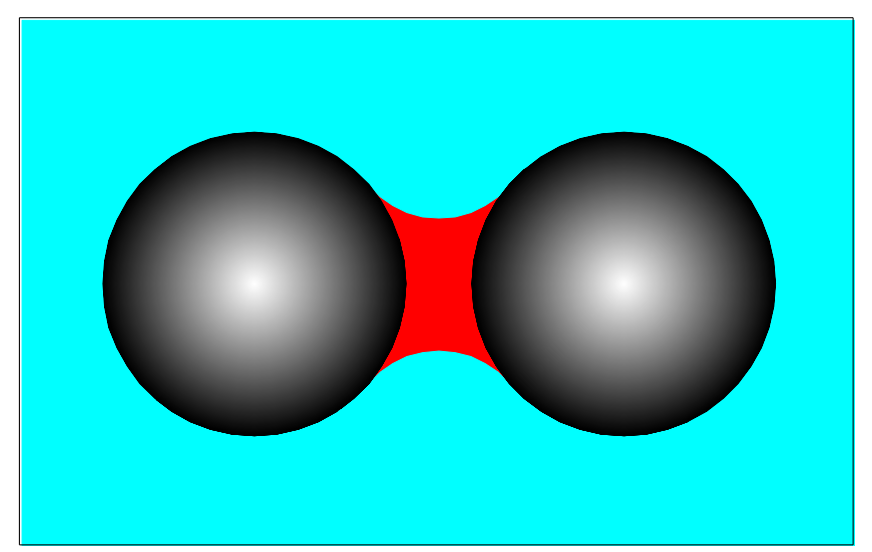




\section{Introduction}

When building a strong sand castle, you should moisten the sand with water. If the sand is too wet, it will be like mud: In contrast, if it is too dry, it will not pack well and simply fall apart. Meanwhile, if you observe carefully the floating matters on the surface of a liquid (e.g., garbage and bubbles floating on the surface of a river), you will find that they tend to gather spontaneously. In the former case moistening the sand causes the capillary bridge force between the sand grains [1-3], while in the latter case self-assembly of the floating matters on the liquid surface is driven by the lateral capillary force [3]. These two types of capillary forces appear not only around us and our natural world, but in a variety of industrial processes. In recent years, the lateral capillary forces have attracted considerable attention in material science and engineering, and are utilized for the formation of two- and three-dimensional arrays of colloid particles.

In the present paper, we overview two methodologies for the computer simulation of wetting and capillary forces as well as particle-stabilized emulsions, which usually appear in a two-phase fluid system including solid bodies. One method is the molecular simulation with a coarse-grained model. The other is the computational fluid dynamics (CFD) with a phase-field model. In Sections 2 and 3, the overview and the applications of the coarse-grained model for molecular simulations are given, while those of the phase-field model for CFD are given in Sections 4 and 5. The relationship between the molecular-scale model and the mesoscale model is briefly explained in Section 4.3. In Section 6, we describe our expected future development of these simulation methods.

\section{Molecular-scale modeling: methods}

\subsection{Molecular simulation}


Molecular simulation is a molecular-scale technique to reproduce in silico (computationally) the motion of a limited number (typically, $10^{2}-10^{6}$ ) of molecules of interest using the molecular dynamics (MD) or the Monte Carlo (MC) method and thereby to estimate the static and dynamic properties of the molecular systems on the basis of statistical mechanics. It is noted that MC gives the static properties only. For details of MD and MC, see Refs. [4-7].

\subsection{Coarse-grained model for molecular systems}

Here, we introduce a coarse-grained molecular model to mimic an oil/water/surfactant system [8-10]. The starting point of this model is to consider two types of particles, that is, hydrophilic particles $(\bullet$; waterlike (w) and headlike (h) particles) and hydrophobic particles (o; oillike (o) and taillike (t) particles). All the molecules are represented by connecting these particles arbitrarily. Several examples of the coarse-grained model are illustrated in Fig. 1 [11-15]: (i) a water molecule consists of one w particle; (ii) an oil molecule consists of one o particle; and (iii) a surfactant molecule is composed of a headgroup of $\mathrm{h}$ particles and a tail chain of $\mathrm{t}$ particles, whose neighboring pairs are connected together. By connecting different number of particles $h$ and $t$ in different arrangement, the coarse-grained model can create surfactants with various molecular architectures. This is the essential idea of the coarsegrained model.

The non-bonded pairs $i$ and $j$ of the particles interact with each other via the truncated Lennard-Jones (12-6) potential with energy parameter $\varepsilon$, size parameter $\sigma$, and cutoff distance $r_{\alpha(i) \alpha(j)}^{\text {cut }}$ :

$$
\left.\begin{array}{l}
u_{\alpha(i) \alpha(j)}^{\mathrm{non}-\mathrm{bond}}\left(r_{i j}\right)= \begin{cases}u_{\mathrm{LJ}}\left(r_{i j}\right)-u_{\mathrm{LJ}}\left(r_{\alpha(i) \alpha(j)}^{\mathrm{cut}}\right), & r_{i j} \leq r_{\alpha(i) \alpha(j)}^{\mathrm{cut}} \\
0, & r_{i j}>r_{\alpha(i) \alpha(j)}^{\mathrm{cut}}\end{cases} \\
\left.\begin{array}{l}
u_{\mathrm{LJ}}\left(r_{i j}\right)=4 \varepsilon\left[\left(\frac{\sigma}{r_{i j}}\right)^{12}-\left.\left(\frac{\sigma}{r_{i j}}\right)^{6}\right|_{\mathrm{w}} ^{\mathrm{c}}\right\rfloor \\
r_{\mathrm{ww}}^{\mathrm{cut}}=r_{\mathrm{hh}}^{\mathrm{cut}}=r_{\mathrm{wh}}^{\mathrm{cut}}=r_{\mathrm{oo}}^{\mathrm{cut}}=r_{\mathrm{tt}}^{\mathrm{cut}}=r_{\mathrm{ot}}^{\mathrm{cut}}=2.5 \sigma
\end{array}\right\} \\
r_{\mathrm{wo}}^{\mathrm{cut}}=r_{\mathrm{ho}}^{\mathrm{cut}}=r_{\mathrm{ht}}^{\mathrm{cut}}=2^{1 / 6} \sigma
\end{array}\right\}
$$


where $r_{i j}$ is the interparticle distance and $\alpha(i)(=\mathrm{w}, \mathrm{h}, \mathrm{o}, \mathrm{t})$ denotes the type of particle $i$. Because the potential of Eq. (1b) exhibits a single well of $-\varepsilon$ at $r_{i j}=2^{1 / 6} \sigma(=1.12 \sigma)$, it give repulsive and attractive interactions for $r_{i j}<2^{1 / 6} \sigma$ and $r_{i j}>2^{1 / 6} \sigma$, respectively. To represent the hydrophilicity and hydrophobicity of the particles (w, h, o, and t), different values of $r_{\alpha(i) \alpha(j)}^{\mathrm{cut}}, 2.5 \sigma$ and $2^{1 / 6} \sigma$ are chosen depending on the types of pairs, as in Eq. (1c): The intraspecies interaction using $r_{\alpha(i) \alpha(j)}^{\mathrm{cut}}=2.5 \sigma$ includes repulsive and attractive forces, whereas the interspecies interaction using $r_{\alpha(i) \alpha(j)}^{\mathrm{cut}}=2^{1 / 6} \sigma$ is completely repulsive.

It is worth noting that the above parameters are a typical set of the parameters used for the coarsegrained model and should be finely tuned depending on the systems and phenomena of interest. The examples are given as follows. First, nonionic and ionic surfactants are frequently modeled using $r_{\mathrm{hh}}^{\mathrm{cut}}=$ $2.5 \sigma$ and $2^{1 / 6} \sigma$ for $\mathrm{h}-\mathrm{h}$ interaction, respectively. In the latter case, only the repulsion exists between particles of the headgroups, which are designated by $\mathrm{H}$ instead of $\mathrm{h}[11,16,17]$ (see also Fig. 1). This is analogous to the electrostatic repulsion between charged headgroups of real ionic surfactants. Second, a binary fluid mixture of water molecules and hydrophobic molecules (e.g., dissolved gaseous molecules and oil molecules) is sometimes modeled using $r_{\mathrm{oo}}^{\mathrm{cut}}=2^{1 / 6}$ instead of $r_{\mathrm{oo}}^{\mathrm{cut}}=2.5 \sigma[18]$. Third, the energy parameter $\varepsilon$ and the size parameter $\sigma$ do not have to be the same for all the particles as in Eq. (1b), but can be tuned depending on the types of the particles (w, h, o, and t) [19-24].

The neighboring particles in a surfactant molecule are connected together by the harmonic potential with the spring length $l_{0}$ and spring constant $k_{l}$ :

$$
u_{\alpha(i) \alpha(j)}^{\mathrm{bond}}\left(r_{i j}\right)=\frac{k_{l}}{2}\left(r_{i j}-l_{0}\right)^{2}
$$

The values of $l_{0}$ and $k_{l}$ must be chosen such that the bonds neither break up nor pass though each other during the course of simulations: typically, $k_{l}=200 \varepsilon / \sigma^{2}$ and $l_{0}=\sigma$ are employed $[13,14]$. This model surfactant is flexible compared with a real surfactant whose conformation is restricted by the bending and torsional angles. Although it is possible to incorporate the potentials of these angles into the model 
surfactant, this additional extension spoils the advantage of the coarse-grained model, that is, the simplicity (e.g., less computational cost and easy coding). If necessary, one should extend this model with considering a trade-off.

Although the coarse-grained model is extremely simplified compared with the real molecules as mentioned above, it can reproduce, at least qualitatively, the essential features of an oil/water/surfactant system [8-10,19,20]:

(i) oil and water hardly mix with each other;

(ii) surfactant molecules are dissolved into oil and water, and spontaneously assemble therein when the surfactant concentration is sufficiently large;

(iii) surfactant molecules adsorb at the oil/water interface and consequently reduce the interfacial tension.

\subsection{Solid surfaces with wettability}

The planar walls and the spherical colloids with the fluid-wettable surface can be modeled by arranging waterlike and/or oillike particles of Eq. (1) into planar and spherical shapes, respectively [2527], though numerous particles are required for this purpose. Alternatively, the smeared-out solid model is highly effective, when the molecular-scale corrugation/roughness of the surface and the compositional heterogeneity, that is, physical and chemical heterogeneities, are not important. In the smeared-out solid model, the interaction between a fluid particle and the solid surface is represented by the (10-4-3) or the (9-3) potential [28,29]. Because the (10-4-3) and the (9-3) potentials show a singlewell profile like the LJ (12-6) potential of Eq. (1b), the hydrophilicity and hydrophobicity of the solid surface for the fluid particles can be represented by choosing their cutoff distance like Eq. (1a) for a fluid-fluid interaction [11]. The direct interaction between the solid surfaces is negligibly smaller than the fluid-induced interaction [12] and therefore is not considered in most of our studies [12-15]. 


\section{Molecular-scale modeling: applications}

\subsection{Hydrophobic colloids in water including a trace amount of inert gaseous molecules}

Let us consider the system, where two hydrophobic colloids are immersed in water including a trace amount of inert gaseous molecules: the hydrogen bond of water-water interaction $(\sim 10 \mathrm{~kJ} / \mathrm{mol})$ is dominant over the van der Waals interactions $(\sim 1 \mathrm{~kJ} / \mathrm{mol})$ between the inert gaseous molecules and between the inert gaseous molecule and the water molecule. Therefore, a water molecule and an inert gaseous molecule were modeled by one w particle and one g particle, respectively, using Eqs. (1a) and (1b) with

$r_{\mathrm{ww}}^{\mathrm{cut}}=2.5 \sigma, \quad r_{\mathrm{gg}}^{\mathrm{cut}}=r_{\mathrm{wg}}^{\mathrm{cut}}=2^{1 / 6} \sigma$

instead of Eq. (1c). To represent the hydrophobic colloids of radius $a=5 \sigma$, the cutoff distance of the potential was chosen such that the fluid-colloid interactions have no attractive forces, but the shortdistance-range repulsive forces originated from the excluded-volume effect. The thermodynamic state of the fluid was set as the reduced density of $\rho^{*}=\rho \sigma^{3}=0.7$ and the reduced temperature of $T^{*}=k_{\mathrm{B}} T / \varepsilon=$ $1.0[13,14]$, where $k_{\mathrm{B}}$ is the Boltzmann constant. The physical quantities of LJ fluids are frequently normalized by the LJ units (energy $\varepsilon$, length $\sigma$, and mass $m$ ) and the resultant dimensionless quantities are referred to as the "reduced" quantities $[4,6,7]$. Henceforth, $X_{\mathrm{g}}$ is defined as the volume fraction of the $\mathrm{g}$ particles in the binary fluid mixture of the $\mathrm{w}$ and $\mathrm{g}$ particles.

In the case of the pure waterlike fluid $\left(X_{\mathrm{g}}=0\right)$, the density profile of fluid near the colloid surface demonstrates that the surface of hydrophobic colloids repelled the waterlike particles toward the bulk fluid region such that no dense layer of the waterlike particles formed anywhere, which is characteristic of dewetting [14]. This should be explained by the speculation that the waterlike particles exist in the bulk fluid more stably than near the colloid surface, since the attractive force of water-water interaction 
is stronger than that of water-colloid interaction in the present system $[12,14]$. Figure 2 a displays the interaction force between the colloids, $F_{\mathrm{pp}}$, as a function of the intersurface distance, $D$, where the force exhibited a monotonic attraction. This result is interpreted as follows: when the colloids approached each other, the unstable waterlike particles next to the colloid surfaces were pushed out into the bulk fluid, by which the total free energy of the system decreased consequently $[12,14]$. In contrast, when the colloid surfaces were hydrophilic, the surface force oscillated with the periodicity of the diameter of a waterlike particle $(\approx \sigma)$ due to the presence of the dense layers of waterlike particles near the hydrophilic surfaces (see Refs. [12,13] and references therein).

Next, we consider the waterlike fluid including a trace amount of inert gaseous particles $\left(X_{\mathrm{g}}=0.003\right.$ and 0.005). As shown in Fig. 3a for $X_{\mathrm{g}}=0.005$, the inert particles were significantly concentrated near the hydrophobic colloid surfaces to form their dense layers, the thickness of which was one-to-two diameters of the particles $(\approx 2 \sigma)$. This should result from the following behavior: the waterlike particles tended to push the inert particles out of the bulk fluid into the fluid-colloid interfacial region, since the presence of the inert particles in the bulk fluid led to the reduction of the attractive interactions among the waterlike particles and therefore was energetically unfavorable. The layer of inert particles near the colloid surface became denser and thicker with increasing the concentration of the inert particles, $X_{\mathrm{g}}$. Parts b and c of Fig. 2 show that the surface forces for $X_{\mathrm{g}}=0.003$ and 0.005 exhibited a monotonically attractive profile in essence, though they had small oscillations with the distance periodicity of the fluid particle diameter $(\approx \sigma)$ near the contact point of $D / \sigma=1$. When the colloids were well separated from each other, the dense layers of inert particles existed independently of each other (see Fig. 3a), resulting in no attractive interaction between them (see Fig. 2c). At the onset of the attractive interaction between the colloids, the dense layers of inert particles fused together to bridge the gap between the colloid surfaces (see Fig. 3b). This is typical of capillary condensation and the accompanying capillary bridge force. As in Fig. 2, the onset distance of the attractive interaction became longer with increasing the concentration of the inert particles, where the layers of inert particles near the colloid surfaces became thicker to merge together at the longer separation distance between the colloids. 
Similar results have been reported by other research groups [18,21]. Similar phenomena will be introduced in Section 5.1.

\subsection{Others}

The other studies of wetting and capillary force using the molecular simulation with the coarsegrained model have been reported as follows: (i) the wettable colloid and plate in unsaturated vapor [15]; (ii) the hydrophilic colloids in binary fluid mixtures of alcohol and water [13,14]; (iii) the colloids with homeotropic anchoring surfaces in a nematic liquid crystal [30]; (iv) the colloids with polymeradsorbed surfaces [31].

\section{Mesoscale modeling: methods}

\subsection{Computational fluid dynamics}

Computational fluid dynamics (CFD) is a meso/macroscale technique to reproduce in silico the dynamic motion of fluids of interest by solving the Navier-Stokes equations or their derivative equations and thereby to estimate the physical quantities such as pressure, velocity, and density of the fluids. CFD has two descriptions, that is, the Eulerian and the Lagrangian descriptions. In the present paper, we introduce the former description, where the physical space of interest is divided into a lattice. The Eulerian CFD is classified into two computational techniques: the direct numerical simulation (DNS) and the lattice Boltzmann method (LBM). For more details of CFD, see Refs. [32-35].

\subsection{Phase-field model for a two-phase fluid}


Let us consider the phase separation in a binary mixture of " $\mathrm{A}$ " and " $\mathrm{B}$ " molecules, where the " $\mathrm{A}$ " rich phase coexists with the "B" rich phase, as illustrated in Fig. 4a. A phase-field model is very useful for simulating the motion of this two-phase flow. In this model, two phases are distinguished by the compositional order parameter, $\psi$, which is a function of position $\mathbf{x}$ in the spatial lattice: the " $\mathrm{A}$ " rich and the "B" rich phases are defined as the regions of $\psi(\mathbf{x})=\psi_{1}$ and $\psi(\mathbf{x})=\psi_{2}\left(\psi_{1}<\psi_{2}\right)$, respectively, whereas the region of the intermediate values of $\psi_{1}<\psi(\mathbf{x})<\psi_{2}$ is considered as the interface between these two phases (see Fig. 4b). It is noted that this order parameter corresponds to the volume fraction of one component for a binary liquid-liquid system or to the mass density of fluid for a single-component vapor-liquid system (i.e., a mixture of "A" phantom molecules and "B” fluid molecules).

The free energy density of the bulk phase without interface, $f_{0}(\psi)$, is given by

$f_{0}(\psi)=-\frac{A}{2} \psi^{2}+\frac{B}{4} \psi^{4}$

where $A$ and $B$ are positive constants. $f_{0}(\psi)$ exhibits a double-well profile shown in Fig. 4c and has two minima at $\psi=\psi_{1}$ and $\psi_{2}$, that is

$f_{0}^{\prime}(\psi)=-A \psi+B \psi^{3}=B \psi\left(\psi-\psi_{1}\right)\left(\psi-\psi_{2}\right)$

$\psi_{1}=-\sqrt{\frac{A}{B}}, \quad \psi_{2}=\sqrt{\frac{A}{B}}$

The free energy functional of the system is obtained by integrating a sum of the bulk-phase free energy density and the interfacial energy density over the volume $V$ composed of small elements $\mathrm{d} \mathbf{x}(=\mathrm{d} x \mathrm{~d} y \mathrm{~d} z)$ :

$$
\begin{aligned}
& F[\psi(\mathbf{x}, t)]=\frac{1}{\beta} \int_{V} f(\psi) \mathrm{d} \mathbf{x} \\
& f(\psi)=f_{0}(\psi)+\frac{K}{2}|\nabla \psi|^{2}
\end{aligned}
$$

where $K$ is a positive constant to determine the interfacial tension and $\beta=1 / k_{\mathrm{B}} T$. Equation (6) is referred to as the Ginzburg-Landau free energy [36,37]. The average composition of the system must be conserved, leading to 


$$
\int_{V} \psi(\mathbf{x}, t) \mathrm{d} \mathbf{x}=\text { const }
$$

\subsection{Relationship between phase-field model and coarse-grained molecular model}

Here, let us briefly explain how the phase-field model is correlated with the coarse-grained molecular model mentioned in Section 2.2. Given that "A" and "B" molecules identical to respectively the oillike and the waterlike particles employed in the coarse-grained model, one can consider the order parameter $\psi$ as the difference in number between the oillike and waterlike particles in a volume element of $\mathrm{dx}$, that

is, the difference in volume fraction between these two species of particles. A summation of the interaction potentials of Eq. (1) over all pairs of the particles in the system and the entropy of mixing are approximated by the free energy of Eq. (6) with the constraint of Eq. (7), where the mean-field approximation and the Ginzburg-Landau expansion near the critical point are employed [37]. The lower limit of the spatial resolution should be $0.1-1 \mathrm{~nm}$ for the coarse-grained molecular model and 10-100 $\mathrm{nm}$ for the phase-field model, because the former is based on the coarse-grained molecules and the latter is on the compositional order parameter. The phase-field model takes no account of the molecular-scale structures of the liquid-liquid and the vapor-liquid interfaces, the actual thickness of which is less than $1 \mathrm{~nm}$.

\subsection{Time-evolution equations for an incompressible two-phase fluid flow}

The order parameter at position $\mathbf{x}$ and at time $t, \psi=\psi(\mathbf{x}, t)$, evolves to minimize the total free energy of the system given by Eq. (6), following

$$
\begin{aligned}
& \frac{\partial \psi}{\partial t}+\mathbf{u} \cdot \nabla \psi=L \nabla^{2}(\beta \mu) \\
& \mu=\frac{\delta F}{\delta \psi}=\frac{1}{\beta}\left[\frac{\partial f}{\partial \psi}-\left.\nabla \cdot \frac{\partial f}{\partial(\nabla \psi)}\right|^{\prime}=\frac{1}{\beta}\left[f_{0}^{\prime}(\psi)-K \nabla^{2} \psi\right]\right.
\end{aligned}
$$


where $L$ is the transport coefficient, $\mathbf{u}$ is the velocity of fluid, and $\mu$ is the local chemical potential. The velocity of an incompressible fluid at position $\mathbf{x}$ and at time $t, \mathbf{u}=\mathbf{u}(\mathbf{x}, t)$, evolves following

$$
\nabla \cdot \mathbf{u}=0
$$

$\rho\left[\frac{\partial \mathbf{u}}{\partial t}+(\mathbf{u} \cdot \nabla) \mathbf{u}\right]_{]}=-\nabla p+\nabla \cdot\left[\eta\left\{\nabla \mathbf{u}+(\nabla \mathbf{u})^{\mathrm{T}}\right\}\right]-\psi \nabla \mu+\rho \mathbf{g}$

where $p$ is the pressure, $\mathbf{g}$ is the body force including the gravity, and the third term of the right-hand side of Eq. (10) stems from the interfacial tension. The physical properties of mass density $\rho$ and viscosity $\eta$ are given by

$$
\begin{aligned}
& \rho=\rho(\mathbf{x}, t)=\frac{\psi(\mathbf{x}, t)-\psi_{1}}{\psi_{2}-\psi_{1}}\left(\rho_{2}-\rho_{1}\right)+\rho_{1} \\
& \eta=\eta(\mathbf{x}, t)=\frac{\psi(\mathbf{x}, t)-\psi_{1}}{\psi_{2}-\psi_{1}}\left(\eta_{2}-\eta_{1}\right)+\eta_{1}
\end{aligned}
$$

where $\rho_{1}$ and $\rho_{2}\left(\eta_{1}\right.$ and $\left.\eta_{2}\right)$ are the density (viscosity) of "A" and "B" rich phases, respectively.

In the research field of statistical physics, a set of Eqs. (8)-(10) is referred to as the model H [36], the original form of which has the additional term of thermal fluctuations for the order parameter and the fluid velocity in the right-hand side of Eqs. (8a) and (10), respectively. Once solving the above equations numerically, one can observe in silico the motion of a two-phase fluid flow.

\subsection{Interfacial profile of a two-phase fluid}

Imagine a system with a half-space of " $A$ " rich phase in coexistence with a half-space of " $B$ " rich phase, where the interface is planar and parallel to the $y z$ plane (perpendicular to the $x$ axis) [37]. Then, one has only to consider a one-dimensional composition variation, $\psi(x)$, with boundary conditions:

$$
\psi(x)= \pm \sqrt{\frac{A}{B}}, \quad \frac{\mathrm{d} \psi}{\mathrm{d} x}=0 \quad \text { at } x= \pm \infty
$$

The function, $\psi(x)$, which minimizes the total free energy, $F$, is determined by the equation of $\mu=0$ 
according to Eq. (8b):

$-A \psi+B \psi^{3}-K \frac{\mathrm{d}^{2} \psi}{\mathrm{d} x^{2}}=0$

When multiplying the both sides of Eq. (13) by $\mathrm{d} \psi / \mathrm{d} x$, one can integrate it as

$-\frac{A}{2} \psi^{2}+\frac{B}{4} \psi^{4}-\frac{K}{2}\left(\frac{\mathrm{d} \psi}{\mathrm{d} x}\right)^{2}=-\frac{A^{2}}{4 B}$

where Eq. (12) is used to determined the right-hand side of Eq. (14). The solution is then

$\psi(x)=\sqrt{\frac{A}{B}} \tanh \left(\sqrt{\frac{A}{2 K}} x\right)$

The profile of Eq. (15) corresponds to the half right-hand side of Fig. 4b. A measure of the width of the interfacial region is given by

$\xi=\sqrt{\frac{2 K}{A}}$

One inserts this solution back into the free energy per unit area and subtracts off that of the bulk phases to find the interfacial tension:

$\gamma=\frac{1}{\beta} \int_{-\infty}^{\infty} \mathrm{d} x\left[f_{0}(\psi)+\frac{K}{2}\left(\frac{\mathrm{d} \psi}{\mathrm{d} x}\right)^{2}-f_{0}(\psi= \pm \sqrt{A / B}) \mid\right.$

Equation (17) with Eqs. (14) and (15) results in

$\gamma=\frac{K}{\beta} \int_{-\infty}^{\infty} \mathrm{d} x\left(\frac{\mathrm{d} \psi}{\mathrm{d} x}\right)^{2}=\frac{(2 A)^{3 / 2} K^{1 / 2}}{3 \beta B}$

\subsection{Solid surfaces with wettability}

Let us explain briefly how to model the fluid-wettable surface of solid bodies. A common technique for this is based on the Cahn model [38], where the free energy of Eq. (6a) has an additional surface term to describe the interactions at the interface between the solid and fluid. The additional term, $F_{\mathrm{S}}$, has a form 


$$
F_{\mathrm{S}}\left[\psi\left(\mathbf{x}_{\mathrm{S}}, t\right)\right]=\frac{1}{\beta} \int_{S}(-H \psi) \mathrm{d} S
$$

where $\mathbf{x}_{\mathrm{S}}$ is the position of solid surface, $S$ is the total area of solid surface, and $H$ is a constant parameter to control the wettability. When $H=0$, the surface exhibits a neutral wettability for both "A" and "B" molecules. In the case of $H<0$, the surface has a strong affinity for "A" molecules, whereas it has a strong affinity for "B" molecules in the case of $H>0$. Thus, one can model the solid surface with arbitrary wettability by tuning the value of $H$ in Eq. (19). It is noted that the order parameters at the solid surfaces, $\psi\left(\mathbf{x}_{\mathrm{S}}, t\right)$, are dynamic variables in the Cahn model of Eq. (19) and hence they must be determined every time step.

Alternatively, one can adopt the same approach as in the coarse-grained molecular model (see Section 2.3). Once a constant value ranging from $\psi_{1}$ and $\psi_{2}$ is pre-assigned to the compositional order parameters at the solid surface, $\psi\left(\mathbf{x}_{\mathrm{S}}, t\right)$, the two-phase fluid near the solid surface should autonomously evolve to minimize the free energy of the system given by Eq. (6a). Indeed, the solid surface of $\psi\left(\mathbf{x}_{\mathrm{S}}, t\right)$ $\equiv \psi_{1}$ and $\psi\left(\mathbf{x}_{\mathrm{S}}, t\right) \equiv \psi_{2}$ attracts the "A" and the "B" rich phases, respectively. When a drop of the "B" rich phase in the "A" rich phase spreads on the physicochemically homogeneous solid surface of $\psi\left(\mathbf{x}_{\mathrm{S}}, t\right)$ $\equiv \psi_{\mathrm{S}}$, the equilibrium contact angle $\theta_{\mathrm{B}, \text { eq }}$ of the drop is given by the following expression [39]:

$$
\begin{aligned}
& \cos \theta_{\mathrm{B}, \mathrm{eq}}=\frac{\chi_{\mathrm{B}}}{2}\left(3-\chi_{\mathrm{B}}^{2}\right) \\
& \chi_{\mathrm{B}}=\frac{\psi_{\mathrm{S}}-\psi_{\mathrm{d}}}{\psi_{2}-\psi_{\mathrm{d}}} \\
& \psi_{\mathrm{d}}=\frac{\psi_{1}+\psi_{2}}{2}
\end{aligned}
$$

where the parameter $\chi_{\mathrm{B}}$ represents the affinity of solid surface for " $\mathrm{B}$ " molecules and satisfies $-1 \leq \chi_{\mathrm{B}} \leq$ 1 (i.e., $\psi_{1} \leq \psi_{\mathrm{S}} \leq \psi_{2}$ ). One can model a solid surface with arbitrary wettability by tuning the parameter $\psi_{\mathrm{S}}\left(\right.$ or $\left.\chi_{\mathrm{B}}\right)$. When $\psi\left(\mathbf{x}_{\mathrm{S}}, t\right) \equiv 0$, the surface has a neutral wettability for both " $\mathrm{A}$ " and "B" molecules: for examples, a liquid drop of "A" or "B" rich phase on this solid surface exhibits the contact angle of $90^{\circ}$ 
and the colloids with this surface strongly attach to the interface between two phases to reduce the interfacial tension, which are referred to as surface-active properties.

In addition to the homogeneous surfaces, one can model the physically and/or chemically heterogeneous surfaces using these two approaches [39,40]. In our model, Eq. (20) is valid for the homogeneous surfaces other than for the heterogeneous surfaces in principle; nevertheless, it can be a measure of the contact angle of a drop on a heterogeneous surface, once the average affinity of the surface is estimated [39].

\subsection{Colloid particles in an incompressible two-phase fluid flow}

Here, let us consider $N$ spherical colloids with radius $a$ suspended in an incompressible two-phase fluid. The continuous concentration field of $N$ colloids at $\left\{\mathbf{X}_{1}, \ldots, \mathbf{X}_{N}\right\}$ is

$$
\begin{aligned}
& \Phi(\mathbf{x}, t)=\sum_{i=1}^{N} \Phi_{i}(\mathbf{x}, t) \\
& \Phi_{i}(\mathbf{x}, t)=g\left(\left|\mathbf{x}-\mathbf{X}_{i}\right|\right)
\end{aligned}
$$

where every colloid has the diffuse interface of thickness $\xi_{\mathrm{p}}(\sim \Delta x)$ and is represented by

$$
\begin{aligned}
& g(r)=\frac{h\left[\left(a+\xi_{\mathrm{p}} / 2\right)-r\right]}{h\left[\left(a+\xi_{\mathrm{p}} / 2\right)-r\right]+h\left[r-\left(a-\xi_{\mathrm{p}} / 2\right)\right]} \\
& h(s)= \begin{cases}\exp \left[-(\Delta x)^{2} / s^{2}\right], & s \geq 0 \\
0, & s<0\end{cases}
\end{aligned}
$$

which was proposed in Ref. [41]. As an alternative to Eq. (22), one can use

$$
g(r)=\frac{1}{2}\left[\tanh \left(\frac{a-r}{\xi_{\mathbf{p}}}\right)+1\right]
$$


which has been frequently used [42,43]. The form of Eq. (23) is equivalent to that of Eq. (15) for interfacial profile of a two-phase fluid. It should be noted that $0 \leq g(r) \leq 1$ for Eq. (22) and $0<g(r)<1$ for Eq. (23). The profile of Eq. (22) is more compact than that Eq. (23), as pointed out in Ref. [41].

Similar to Eq. (6), the free energy functional for the fluid domain $(1-\Phi)$ and colloid domain $(\Phi)$ is given by

$$
F[\psi, \Phi]=\frac{1}{\beta} \int_{V} \mathrm{~d} \mathbf{x}\left[f_{0}(\psi)+\frac{K}{2}|\nabla \psi|^{2}+\left.\frac{K_{\mathrm{p}}}{2}\left(\psi-\psi_{\mathrm{p}}\right)^{2} \Phi\right|_{]}\right.
$$

where the additional third term in the integral describes the fluid-wettable surface of colloids: $\psi_{\mathrm{p}}$ is a constant parameter to control the wettability, and $K_{\mathrm{p}}$ is a positive parameter to constrain the compositional order parameter inside the colloids to be $\psi_{\mathrm{p}}$ and must be chosen as a large value compared with $A$ and $B$ appeared in Eq. (4) such that the free energy density inside the colloids without interface, $f_{0}(\psi)+K_{\mathrm{p}}\left(\psi-\psi_{\mathrm{p}}\right)^{2} / 2$, exhibits a single-well profile with a minimum at $\psi \approx \psi_{\mathrm{p}}$. It is noted that Eq. (24) is apparently similar to Eq. (2) of Ref. [44] and their difference is a choice of the models for wettability: the former is based on our idea [39], whereas the latter adopts the Cahn model [38]. Following Eq. (8b), the local chemical potential is calculated as

$\mu=\frac{1}{\beta}\left[f_{0}^{\prime}(\psi)-K \nabla^{2} \psi+K_{\mathrm{p}}\left(\psi-\psi_{\mathrm{p}}\right) \Phi\right]$

Like a set of Eqs. (8)-(10), the time evolutions of the concentration and velocity fields, $\psi$ and $\mathbf{u}$, for the incompressible two-phase fluid including wettable colloids are described by

$$
\nabla \cdot \mathbf{u}=0
$$

$\frac{\partial \psi}{\partial t}+\mathbf{u} \cdot \nabla \psi=L \nabla \cdot\left[\left(\mathbf{I}-\mathbf{n}_{\mathrm{p}} \mathbf{n}_{\mathrm{p}}\right) \nabla(\beta \mu)\right]$

$\rho\left[\frac{\partial \mathbf{u}}{\partial t}+(\mathbf{u} \cdot \nabla) \mathbf{u}\right]_{]}=-\nabla p+\nabla \cdot\left[\eta\left\{\nabla \mathbf{u}+(\nabla \mathbf{u})^{\mathrm{T}}\right\}\right]-\psi \nabla \mu+\rho \mathbf{g}+\mathbf{f}_{\mathrm{p}}$

where $\mathbf{I}$ is the unit tensor, and $\mathbf{n}_{\mathrm{p}}=-\nabla \Phi /|\nabla \Phi|$ is a surface-normal-vector field of $N$ colloids. The operator $\left(\mathbf{I}-\mathbf{n}_{\mathrm{p}} \mathbf{n}_{\mathrm{p}}\right)$ ensures the conservation of $\psi$, because the no-penetration condition of $\mathbf{n}_{\mathrm{p}} \cdot \nabla(\beta \mu)=0$ 
is directly assigned at the diffuse interface; this idea was originally proposed for charged colloids in electrolytes [45]. The force field $\mathbf{f}_{\mathrm{p}}$ stemming from colloids is given by

$$
\begin{aligned}
& \mathbf{f}_{\mathrm{p}}=\mathbf{f}_{\mathrm{p}}(\mathbf{x}, t)=\sum_{i=1}^{N}\left(\mathbf{F}_{i}^{\mathrm{p}}+\mathbf{F}_{i}^{\mathrm{pp}}\right) \frac{\Phi_{i}(\mathbf{x}, t)}{v_{i}} \\
& v_{i}=\int_{V} \Phi_{i}(\mathbf{x}, t) \mathrm{d} \mathbf{x} \approx \frac{4 \pi}{3} a^{3}
\end{aligned}
$$

where $v_{i}$ is the volume of $i$-th spherical colloid, $\mathbf{F}_{i}^{\mathrm{p}}$ is the external force (except the gravity) acting on $i$ th colloid, and $\mathbf{F}_{i}^{\mathrm{pp}}$ is the force on $i$-th colloid interacting with other colloids:

$$
\begin{aligned}
& \mathbf{F}_{i}^{\mathrm{pp}}=-\frac{\partial E^{\mathrm{pp}}}{\partial \mathbf{X}_{i}} \\
& E^{\mathrm{pp}}=E^{\mathrm{pp}}\left(\mathbf{X}_{1}, \ldots, \mathbf{X}_{N}\right)=\sum_{i=1}^{N-1} \sum_{j=i+1}^{N} E_{i j}^{\mathrm{pp}}\left(\left|\mathbf{X}_{i}-\mathbf{X}_{j}\right|\right) \\
& E_{i j}^{\mathrm{pp}}\left(R_{i j}\right)=\left\{\begin{array}{l}
\left.\infty,\left[\frac{\sigma}{R_{i j}-2 a}\right)^{12}-\left.\left(\frac{\sigma}{R_{i j}-2 a}\right)^{6}\right|^{6}\right\rfloor, \quad 2 a<R_{i j} \leq 2 a+2^{1 / 6} \sigma \\
(0,
\end{array}\right. \\
& R_{i j}=\left|\mathbf{X}_{i}-\mathbf{X}_{j}\right|
\end{aligned}
$$

where $\varepsilon$ and $\sigma$ should be taken as $\varepsilon \propto a^{n}(n=1$ or 2$)$ and $2^{1 / 6} \sigma \geq \xi_{\mathrm{p}}$, respectively, such that the colloids with the diffuse interface, the outermost radius of which is equal to $a+\xi_{\mathrm{p}} / 2$ because of Eq. (22), never overlap with each other.

Mass density $\rho$ and viscosity $\eta$ of the simulation domain are given by

$$
\begin{aligned}
& \rho=\rho(\mathbf{x}, t)=[1-\Phi(\mathbf{x}, t)] \rho_{\mathrm{f}}+\Phi(\mathbf{x}, t) \rho_{\mathrm{p}} \\
& \eta=\eta(\mathbf{x}, t)=[1-\Phi(\mathbf{x}, t)] \eta_{\mathrm{f}}+\Phi(\mathbf{x}, t) \eta_{\mathrm{p}}
\end{aligned}
$$

respectively, where $\rho_{\mathrm{f}}$ and $\eta_{\mathrm{f}}$ for the fluid domain are given by Eqs. (11a) and (11b), respectively. In contrast, the setting of $\rho_{\mathrm{p}}$ and $\eta_{\mathrm{p}}$ for the colloid domain depends on the techniques for tracking the 
motion of the colloids, which include the fluid particle dynamics (FPD) method [42], the smoothed profile (SP) method [41], and the others [43].

Hereafter, we employ the FPD method [42], where a colloidal suspension is treated as the undeformable but viscous fluid particles with the viscosity $\eta_{\mathrm{p}}$ suspended in a fluid domain with the viscosity $\eta_{\mathrm{f}}$. In the limit of $\eta_{\mathrm{p}} / \eta_{\mathrm{f}} \rightarrow \infty$, the fluid particles can be regarded as solid ones. This viscosity ratio is a measure of the accuracy of this FPD approximation [42]. Practically, one should set as

$\eta_{\mathrm{p}} \approx 50 \times \max \left(\eta_{1}, \eta_{2}\right)$

The translational and angular velocities of $i$-th colloid, $\mathbf{V}_{i}$ and $\boldsymbol{\Omega}_{i}$, are then calculated as

$$
\begin{aligned}
& \frac{\mathrm{d} \mathbf{X}_{i}}{\mathrm{~d} t}=\mathbf{V}_{i}=\frac{1}{v_{i}} \int_{V} \mathrm{~d} \mathbf{x}\left[\mathbf{u}(\mathbf{x}, t) \Phi_{i}(\mathbf{x}, t)\right] \\
& \frac{\mathrm{d} \boldsymbol{\Theta}_{i}}{\mathrm{~d} t}=\mathbf{\Omega}_{i}=\frac{\rho_{\mathrm{p}}}{I_{i}} \int_{V} \mathrm{~d} \mathbf{x}\left\{\left(\mathbf{x}-\mathbf{X}_{i}\right) \times\left[\mathbf{u}(\mathbf{x}, t)-\mathbf{V}_{i}\right] \Phi_{i}(\mathbf{x}, t)\right\}
\end{aligned}
$$

The mass and the principal moment of inertia for $i$-th spherical colloid, $M_{i}$ and $I_{i}$, are respectively given by

$$
\begin{aligned}
& M_{i}=\rho_{\mathrm{p}} \int_{V} \Phi_{i}(\mathbf{x}, t) \mathrm{d} \mathbf{x}=\rho_{\mathrm{p}} v_{i} \approx \frac{4 \pi}{3} \rho_{\mathrm{p}} a^{3} \\
& I_{i}=\rho_{\mathrm{p}} \int_{V} \mathrm{~d} \mathbf{x}\left[\left|\mathbf{x}-\mathbf{X}_{i}\right|^{2} \Phi_{i}(\mathbf{x}, t)\right] \approx \frac{8 \pi}{15} \rho_{\mathrm{p}} a^{5}
\end{aligned}
$$

The position and orientation of $i$-th colloid, $\mathbf{X}_{i}$ and $\Theta_{i}$, are obtained by integrating Eq. (31) numerically.

\section{Mesoscale modeling: applications}

\subsection{Capillary bridge forces between wetting colloids in vapor}

Let us consider the mechanically equilibrated system with no gravity, where two wetting cylinders of radius $a=64.0 \Delta x$ covered with liquid films of thickness $5 \Delta x$ were placed in vapor. Here, $\Delta x$ is the 
lattice spacing and the density ratio of the liquid and vapor phases, $\rho_{\mathrm{L}} / \rho_{\mathrm{V}}$, was set at 5 . Following Eq. (20), the wettability of the cylinders was chosen as $\chi_{\mathrm{L}}=1$ so that the equilibrium contact angle of a liquid drop thereon was equal to $0^{\circ}$. The results were obtained from our LBM simulations [46].

Typical snapshots of the fluid densities are displayed in Fig. 5. The interaction force per unit length between these two cylinders is shown in Fig. 6 as a function of the intersurface distance $D$, where $\gamma_{\text {LV }}$ is the interfacial tension between the liquid and vapor. When the cylinders were well separated from each other, the liquid films adsorbed on the cylinder surfaces existed independently of each other (see Fig. 5a), resulting in the zero interaction forces at the distances of $D \geq 15 \Delta x$ (see Fig. 6). When the two cylinders came closer, the force suddenly became attractive at $D=14 \Delta x$. At the onset of this attraction, the liquid films fused together to bridge the gap between the cylinders (see Fig. 5b). This attraction is referred to as the capillary bridge force. The phenomena mentioned here (Figs. 5 and 6) are essentially the same as those in Section 3.1 (Figs. 2 and 3), although there are the differences in bulk/bridge phases between the former and the latter. These capillary-induced phase transition and accompanying capillary bridge force have been frequently found in the experiments as well as our daily life [2].

\subsection{Lateral capillary forces between colloids at liquid-vapor interface}

Let us consider the mechanically equilibrated system with gravity, where two horizontal wetting cylinders of radius $a=7.9 \Delta x$ were placed at a liquid-vapor interface. The density ratio of the liquid and vapor phases, $\rho_{\mathrm{L}} / \rho_{\mathrm{V}}$, was set at 5 . The results were obtained from our LBM simulations [46]. The snapshots of the fluid densities shown in Fig. 7 illustrate that the liquid surface near the cylinders was deformed, depending on the wettablities of the cylinder surfaces: the wetting cylinders pulled up the liquid surface, while the nonwetting cylinders pushed it down. Here, the wetting and nonwetting cylinders had $\chi_{\mathrm{L}}=0.5$ and -0.5 (i.e., $\theta_{\mathrm{L}, \mathrm{eq}}=47^{\circ}$ and $133^{\circ}$ ), respectively [see Eq. (20)]. 
The interaction forces per unit length between these two cylinders are shown in Fig. 8 as a function of the intersurface distance $D$. The forces between a pair of the wetting or the nonwetting cylinders were attractive, while those between the wetting and nonwetting cylinders were repulsive. These forces are referred to as lateral capillary force, which is clearly distinguished from capillary bride force mentioned in Sections 3.1 and 5.1. As in Fig. 8, the lateral capillary forces between these two cylinders became stronger with decreasing the intersurface distance. The force-distance profiles obtained from our simulation reasonably agreed with those from the theoretical prediction. It should be noted that an interface between two phases represented by the phase-field model is diffuse rather than sharp, as

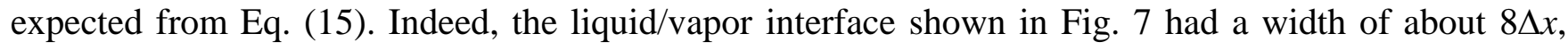
where $\xi=1.8 \Delta x$ was obtained according to Eqs. (15) and (16) [39]. This explains the reason why the interaction forces of our simulation shown in Fig. 8 deviated from those of the theoretical prediction at $D<20 \Delta x$, although our LBM enabled us to estimate them at the distances down to $D=2.6 \Delta x$ [46]. The effects of the interfacial tension and the gravitational acceleration on the lateral capillary forces were also investigated in our study [47].

One can utilize the lateral capillary force as a driving force in order to form two-dimensional arrays of colloid particles at air/liquid interfaces, liquid/liquid interfaces, and solid surfaces.

\subsection{Oil drop covered with surface-active colloids in water under shear}

As far as the two-phase fluids including the immobile colloids are concerned, one has only to solve numerically the equations of motion for the fluids other than for the colloids, the effects of which are considered via the boundary conditions (e.g., no-slip condition) as well as the wettability of the colloid surfaces (see Section 4.6). This is the case for Sections 5.1 and 5.2, in which LBM was employed. Hereafter, we consider the mobile colloids in a liquid-liquid mixture, modeled by the techniques explained in Section 4.7, where we employed DNS other than LBM.

A set of the parameters was chosen as $A=B=K_{\mathrm{p}}=2, K=0.5, L=2, \rho_{\mathrm{f}}\left(=\rho_{1}=\rho_{2}\right)=\rho_{\mathrm{p}}=1, \eta_{\mathrm{f}}\left(=\eta_{1}\right.$ 
$\left.=\eta_{2}\right)=1, \eta_{\mathrm{p}}=50, a=3, \xi_{\mathrm{p}}=2, \varepsilon=0.173$, and $\sigma=2$, where $\xi=0.7 \Delta x$ was obtained from Eq. (16). The equations of motion for the fluid and the colloid particles in a two-dimensional space were solved by the finite-difference schemes with the staggered grids, where the lattice space of $\Delta x=1$ and the time increment of $\Delta t=0.01$ were used. A hybrid scheme of the MAC (Marker-and-Cell) $[48,49]$ and the SMAC (Simplified MAC) methods [50,51] was employed for the time-evolution of Eq. (26c), where the Poisson's equations for the pressure-velocity coupling of Eqs. (26a) and (26c) were solved by the BiCGSTAB (Bi-Conjugate Gradient STABilized) method [52]. The type-B CIP (Cubic-Interpolated Propagation or Constrained Interpolation Profile) scheme [53] and the explicit Euler scheme were used for the time-evolution of Eq. (26b) and Eq. (31), respectively. More details of our DNS technique will be given elsewhere.

As shown in Fig. 9a, a single oil drop $\left(\psi_{1}=-1\right)$ of radius $a_{\text {drop }}=20$ was covered with $N=10$ surfaceactive colloids of $a=3$ and $\psi_{\mathrm{p}}=0$, and was placed in water $\left(\psi_{2}=1\right)$, which filled in the simulation region of $250 \Delta x \times 100 \Delta x$. The upper and lower walls horizontally moved at the velocity of 0.5 and -0.5 , respectively, and the periodic boundary conditions were applied to the horizontal directions. This Pickering emulsion deformed more significantly with time, as shown in Fig. 9 (see also Video Data). The degree of this deformation was influenced not only by the number of the surface-active colloids but also by their initial position: the former determines the reduction of interfacial tension of the emulsion, whereas the latter influences the drag force acting on the colloids by shear.

Similar CFD studies have been reported by other research groups [44,54-57].

\section{Concluding remarks}

In the present paper, we overview two methodologies for the computer simulation of wetting and capillary forces as well as particle-stabilized emulsions. As for molecular simulations based on the coarse-grained model overviewed in Section 2, the main focus has been on the interactions between 
physicochemically homogeneous, undeformable solid surfaces immersed in simple fluids rather than complex fluids. In the immediate future, the vigorous efforts should be focused on complex fluids containing amphiphiles [13,14,23] and polymers [31], interfaces between different phases (e.g., liquid-liquid and liquid-vapor interfaces containing amphiphilic molecules), colloid particles with the physicochemically heterogeneous surfaces [22,23,27], and soft deformable particles, where one should explore not only the interaction forces between the particles in the complex fluids but also the static and dynamics behaviors of these particle suspensions from a molecular point of view. These simulation studies become increasingly important for the handling and processing of advanced, functionalized particulate materials with high accuracy.

Because of the computational cost, however, the molecular dynamics technique using individual molecules is limited to the short-time simulations of small systems, which hardly address the industrially fundamental issues such as the collective behavior of the colloids suspended in complex fluids and the effects of flows on it. For this reason, the CFD with the phase-field model overviewed in Section 4 is very effective and useful as one of the mesoscale techniques to address the issues as well as those mentioned in Section 5. A better understanding of the nonequilibrium phenomena of complex fluids containing colloid particles, in which "interfaces" and "flows" play a crucial role, will give the guidelines of fundamental importance for highly precise and efficient manufacturing processes of industrial products, such as composite materials, composite particles, cosmetics, food, and pharmaceutical products.

\section{Acknowledgements}

The author thanks the editorial board of the Society of Powder Technology, Japan for giving him an opportunity to publish the present review paper that is enlarged from the original Japanese paper [58]. He also thanks for financial support by the Ministry of Education, Culture, Sports, Science and 
Technology (MEXT) in Japan (Grants-in-Aid for Scientific Research, No. 23656489; Strategic Development of Research Infrastructure for Private Universities, No. S0901039), the Japan Society for the Promotion of Science (JSPS; Core-to-Core Program, No. 18004), and Hosokawa Powder Technology Foundation. Our CFD study given in Section 5.3 was contributed in part by Mr. Daisuke Komiyama who wrote the preliminary source code for DNS of two-phase fluid flows. 


\section{Nomenclature}

A parameter for free energy of bulk phase

$\left(\mathrm{m}^{-3}\right)$

a radius of colloid or droplet

$B$ parameter for free energy of bulk phase

D intersurface distance between colloids

E interaction potential between colloids

$F \quad$ total free energy of system

$F_{\mathrm{pp}} \quad$ interaction force between colloids

$\left(\mathrm{N}, \mathrm{N} \mathrm{m}^{-1}\right)$

F force on colloid

$f \quad$ free energy density

$f_{0} \quad$ free energy density of bulk phase

$\left(\mathrm{m}^{-3}\right)$

f force field

$g$ profile of colloid with diffuse interface

g body force acting on fluid

$H \quad$ parameter for wetting energy

I principal moment of inertia for colloid

$\left(\mathrm{kg} \mathrm{m}^{2}\right)$

I unit tensor

$K \quad$ parameter for interfacial energy

$K_{\mathrm{p}} \quad$ parameter for fluid-wettable surface of colloid

$\left(\mathrm{m}^{-3}\right)$

$k_{\mathrm{B}} \quad$ Boltzmann constant

$\left(\mathrm{J} \mathrm{K}^{-1}\right)$

$k_{l} \quad$ spring constant for harmonic potential

$\left(\mathrm{J} \mathrm{m}^{-2}\right)$

$L \quad$ transport coefficient

$\left(\mathrm{m}^{5} \mathrm{~s}^{-1}\right)$

$l_{0} \quad$ spring length for harmonic potential

M mass of colloid

$m \quad$ mass of LJ particle

$N \quad$ number of colloids

n surface-normal-vector field of colloids

$p \quad$ pressure of fluid 
$R \quad$ center-to-center distance between colloids

$r \quad$ distance between fluid particles

$S \quad$ total area of solid surface

$\left(\mathrm{m}^{2}\right)$

$T \quad$ temperature

$t \quad$ time

$u \quad$ interaction potential between fluid particles

u velocity of fluid

$\left(\mathrm{m} \mathrm{s}^{-1}\right)$

$V \quad$ total volume of system

$\left(\mathrm{m}^{3}\right)$

V velocity of colloid $\left(\mathrm{m} \mathrm{s}^{-1}\right)$

$v \quad$ volume of colloid

X volume fraction of fluid component

$\mathbf{X} \quad$ position of colloid

$\mathbf{x} \quad$ position of lattice node

$x, y, z \quad$ Cartesian coordinates

$\beta \quad 1 / k_{\mathrm{B}} T$

$\chi \quad$ affinity of solid surface for fluid molecules

$\gamma \quad$ interfacial tension

$\left(\mathrm{J} \mathrm{m}^{-2}\right)$

$\dot{\gamma} \quad$ shear rate

$\Delta x \quad$ lattice spacing

$\varepsilon \quad$ energy parameter for LJ potential

$\Phi \quad$ concentration field of colloid(s)

$\eta \quad$ viscosity

$\mu \quad$ local chemical potential

$\left(\mathrm{J} \mathrm{m}^{-3}\right)$

$\Theta \quad$ orientation of colloid

$\rho \quad$ number density or mass density

$\left(\mathrm{m}^{-3}, \mathrm{~kg} \mathrm{~m}^{-3}\right)$

$\sigma \quad$ size parameter for $\mathrm{LJ}$ potential

$\mathbf{\Omega} \quad$ angular velocity of colloid

$\left.(\operatorname{rad~s})^{-1}\right)$

$\xi \quad$ interfacial thickness

$\psi \quad$ compositional order parameter 
Super-scripts and sub-scripts

f fluid

g inert gaseous particle

h headlike hydrophilic particle of amphiphile

$i, j \quad$ index of fluid particle or colloid

o oillike particle

p colloid particle

S surface of solid body

$\mathrm{T}$ transverse

t taillike hydrophobic particle of amphiphile

w waterlike particle

$\alpha(i) \quad$ species of fluid particle $i$

* $\quad$ reduced quantity for LJ fluid 
Fig. 1. Coarse-grained model for the molecular simulation of an oil/water/surfactant system including spherical colloids.

Fig. 2. Interaction forces between two hydrophobic colloids of radius $a=5 \sigma$ as a function of the intersurface distance $D$ in a waterlike fluid containing trace amounts of inert gaseous particles at the volume fraction of $X_{\mathrm{g}}=0(\mathrm{a}), 0.003(\mathrm{~b})$, and 0.005 (c).

Fig. 3. Contour maps of the density of inert particles near the hydrophobic colloids for the intersurface distance of $D / \sigma=8.0$ (a) and 5.8 (b) in a waterlike fluid containing inert particles at $X_{\mathrm{g}}=0.005$. Six colors from white to black indicate a region of densities $0-10,10-20,20-30,30-40,40-50$, and more than 50, respectively, which are normalized such that the value of 1 indicates the density in the bulk. The dashed lines depict the surface of the hydrophobic colloids. See also Fig. 2c.

Fig. 4. (a) Illustration of a two-phase fluid system, (b) the cross section of the compositional order parameter profile of panel a, and (c) the free energy density of the bulk phase as a function of the compositional order parameter.

Fig. 5. Snapshots of two wetting cylinders of radius $a=64.0 \Delta x$ covered with liquid films of thickness $5 \Delta x$ in vapor at the intersurface distance of $D=15 \Delta x$ (a) and $14 \Delta x$ (b). Redrawn from Ref. [46].

Fig. 6. Interaction force between two wetting cylinders covered with liquid films in vapor as a function of the intersurface distance $D$. See also Fig. 5. The line is a guide for the eyes. Redrawn from Ref. [46].

Fig. 7. Snapshots of two horizontal cylinders of radius $a=7.9 \Delta x$ and different surface wettabilities, which are separated by the intersurface distance $D=32.2 \Delta x$ and trapped at a liquid-vapor interface in a gravitational field: (A) two wetting cylinders; (B) two nonwetting cylinders; (C) wetting and nonwetting cylinders. Redrawn from Ref. [46].

Fig. 8. Interaction forces between two horizontal cylinders trapped at a liquid-vapor interface in a gravitational field as a function of the intersurface distance $D$ : (A) two wetting cylinders; (B) two 
nonwetting cylinders; (C) wetting and nonwetting cylinders. See also Fig. 7. The lines indicate the theoretical predictions. Redrawn from Ref. [46].

Fig. 9. Snapshots of a single oil drop (blue color) covered with 10 surface-active colloids (red color) in water (green color) under shear at normalized time of $t \dot{\gamma}=0$ (a), 3.0 (b), 4.5 (c), and 6.0 (d). See also Video Data. (For interpretation of the references to color in figure legend, the reader is referred to the web version of the article.) 


\section{References}

[1] J.N. Israelachvili, Intermolecular and Surface Forces, 3rd edn., Academic Press, San Diego, 2010.

[2] D.F. Evans, H. Wennerström, The Colloidal Domain: where Physics, Chemistry, and Biology Meet, 2nd edn., Wiley-VCH, New York, 1999.

[3] P.A. Kralchevsky, K. Nagayama, Particles at Fluid Interfaces and Membranes: Attachment of Colloid Particles and Proteins to Interfaces and Formation of Two-Dimensional Arrays, Elsevier, Amsterdam, 2001.

[4] M.P. Allen, D.J. Tildesley, Computer Simulation of Liquids, Clarendon Press, Oxford, 1987.

[5] A.R. Leach, Molecular Modelling: Principles and Applications, 2nd edn., Prentice Hall, Dorset, 2001.

[6] D. Frenkel, B. Smit, Understanding Molecular Simulation: From Algorithms to Applications, 2nd edn., Academic Press, San Diego, 2002.

[7] D.C. Rapaport, The Art of Molecular Dynamics Simulation, 2nd edn., Cambridge University Press, Cambridge, 2004.

[8] M.M. Telo da Gama, K.E. Gubbins, Mol. Phys., 59 (1986) 227-239.

[9] B. Smit, Phys. Rev. A, 37 (1988) 3431-3433.

[10] B. Smit, P.A.J. Hilbers, K. Esselink, L.A.M. Rupert, N.M. van Os, A.G. Schlijper, J. Phys. Chem., 95 (1991) 6361-6368.

[11] H. Shinto, S. Tsuji, M. Miyahara, K. Higashitani, Langmuir, 15 (1999) 578-586.

[12] H. Shinto, M. Miyahara, K. Higashitani, J. Colloid Interface Sci., 209 (1999) 79-85.

[13] H. Shinto, M. Miyahara, K. Higashitani, Langmuir, 16 (2000) 3361-3371.

[14] H. Shinto, D. Iwahara, M. Miyahara, K. Higashitani, Langmuir, 18 (2002) 4171-4178.

[15] H. Shinto, K. Uranishi, M. Miyahara, K. Higashitani, J. Chem. Phys., 116 (2002) 9500-9509.

[16] B. Smit, K. Esselink, P.A.J. Hilbers, N.M. van Os, L.A.M. Rupert, I. Szleifer, Langmuir, 9 (1993) 9-11.

[17] B. Smit, P.A.J. Hilbers, K. Esselink, Tenside Surf. Det., 4 (1993) 287-293.

[18] M. Kinoshita, J. Chem. Phys., 118 (2003) 8969-8981.

[19] D.R. Rector, F. van Swol, J.R. Henderson, Mol. Phys., 82 (1994) 1009-1031.

[20] M. Kinoshita, Y. Sugai, J. Comput. Chem., 23 (2002) 1445-1455.

[21] H. Greberg, G.N. Patey, J. Chem. Phys., 114 (2001) 7182-7188.

[22] S.D. Overduin, G.N. Patey, J. Chem. Phys., 117 (2002) 3391-3397.

[23] S.D. Overduin, G.N. Patey, J. Chem. Phys., 119 (2003) 8676-8685. 
[24] C.J. Hemming, G.N. Patey, J. Chem. Phys., 121 (2004) 6508-6517.

[25] M. Schoen, D.J. Diestler, J.H. Cushman, J. Chem. Phys., 87 (1987) 5464-5476.

[26] M. Schoen, D.J. Diestler, Phys. Rev. E, 56 (1997) 4427-4440.

[27] Y. Qin, K.A. Fichthorn, J. Chem. Phys., 119 (2003) 9745-9754.

[28] W.A. Steele, Surf. Sci., 36 (1973) 317-352.

[29] W.A. Steele, The interaction of gases with solid surfaces, Pergamon Press, Oxford, 1974.

[30] E.B. Kim, O. Guzman, S. Grollau, N.L. Abbott, J.J. de Pablo, J. Chem. Phys., 121 (2004) 19491961.

[31] K.T. Marla, J.C. Meredith, Langmuir, 21 (2004) 487-497.

[32] T.J. Chung, Computational Fluid Dynamics, 2nd edn., Cambridge University Press, New York, 2010.

[33] S. Succi, The Lattice Boltzmann Equation for Fluid Dynamics and Beyond, Clarendon Press, Oxford, 2001.

[34] G.R. Liu, M.B. Liu, Smoothed Particle Hydrodynamics: A Meshfree Particle Method, World Scientific Publishing, Singapore, 2003.

[35] A. Prosperett, G. Tryggvason (Eds.), Computational Methods for Multiphase Flow, Cambridge University Press, Cambridge, 2007.

[36] P.C. Hohenberg, B.I. Halperin, Rev. Mod. Phys., 49 (1977) 435-479.

[37] S. Safran, Statistical Thermodynamics of Surfaces, Interfaces, and Membranes, Addison-Wesley, Reading, 1994.

[38] J.W. Cahn, J. Chem. Phys., 66 (1977) 3667-3672.

[39] D. Iwahara, H. Shinto, M. Miyahara, K. Higashitani, Langmuir, 19 (2003) 9086-9093.

[40] A. Dupuis, J.M. Yeomans, Langmuir, 21 (2005) 2624-2629.

[41] Y. Nakayama, R. Yamamoto, Phys. Rev. E, 71 (2005) 036707.

[42] H. Tanaka, T. Araki, Phys. Rev. Lett., 85 (2000) 1338-1341.

[43] M. Fujita, Y. Yamaguchi, Phys. Rev. E, 77 (2008) 026706.

[44] T. Araki, H. Tanaka, Phys. Rev. E, 73 (2006) 061506.

[45] Y. Nakayama, K. Kim, R. Yamamoto, Euro. Phys. J. E: Soft Matter Biol. Phys., 26 (2008) 361-368.

[46] H. Shinto, D. Komiyama, K. Higashitani, Adv. Powder Technol., 18 (2007) 643-662.

[47] H. Shinto, D. Komiyama, K. Higashitani, Langmuir, 22 (2006) 2058-2064.

[48] F.H. Harlow, J.E. Welch, Phys. Fluids, 8 (1965) 2182-2189.

[49] J.E. Welch, F.H. Harlow, J.P. Shannon, B.J. Daly, Los Alamos Scientific Laboratory report, LA3425 (1966).

[50] A.A. Amsden, F.H. Harlow, J. Comput. Phys., 6 (1970) 322-325.

[51] A.A. Amsden, F.H. Harlow, Los Alamos Scientific Laboratory report, LA-4370 (1970). 
[52] H.A. van der Vorst, SIAM J. Sci. Stat. Comput., 13 (1992) 631-644.

[53] T. Aoki, CFD J., 4 (1995) 279-291.

[54] K. Stratford, R. Adhikari, I. Pagonabarraga, J.-C. Desplat, M.E. Cates, Science, 309 (2005) 21982201.

[55] A.S. Joshi, Y. Sun, Phys. Rev. E, 79 (2009) 066703.

[56] F. Jansen, J. Harting, Phys. Rev. E, 83 (2011) 046707.

[57] P.C. Millett, Y.U. Wang, J. Colloid Interface Sci., 353 (2011) 46-51.

[58] H. Shinto, J. Soc. Powder Technol., Japan, 46 (2009) 25-34. 


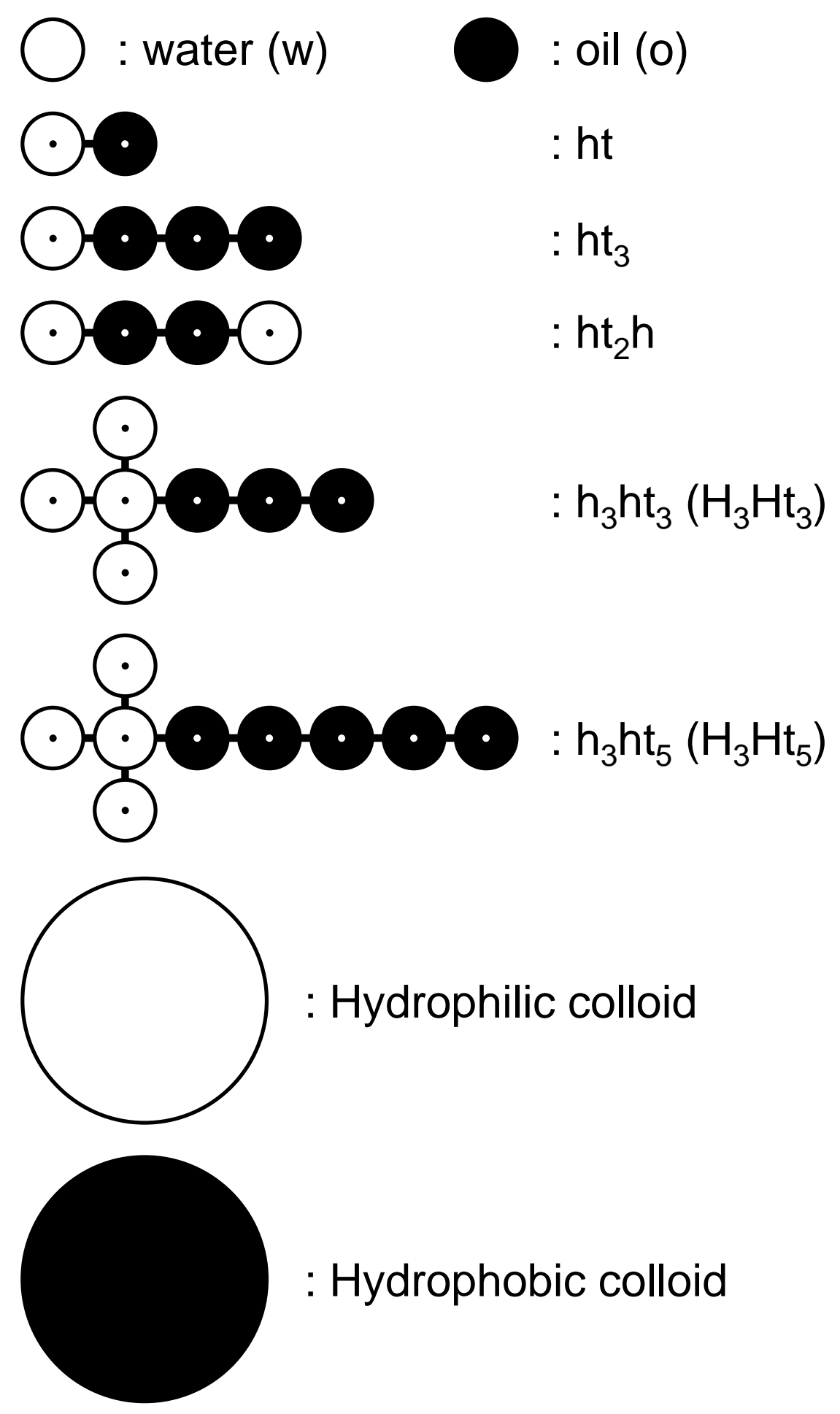


Fig. 2

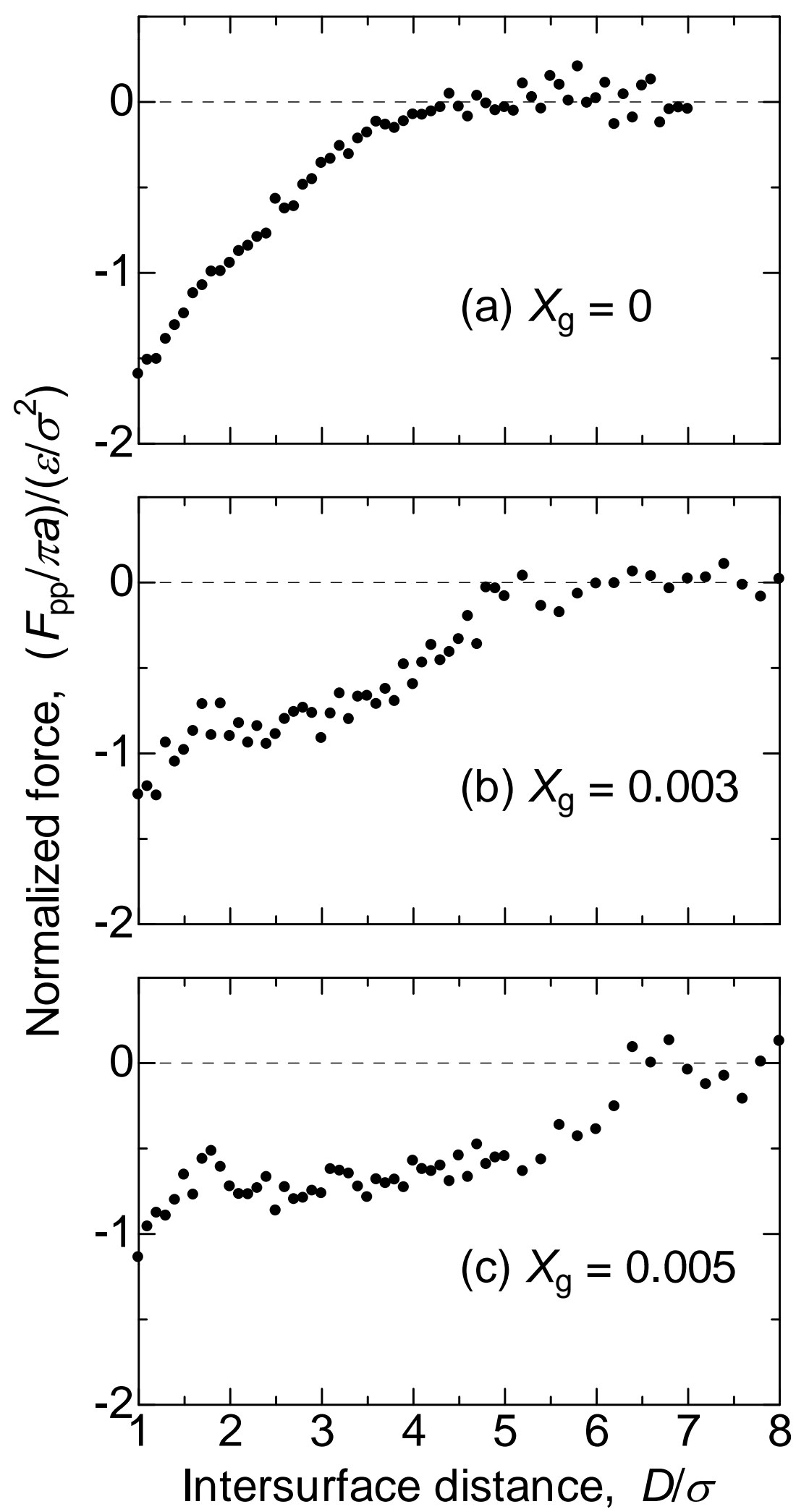


Fig. 3
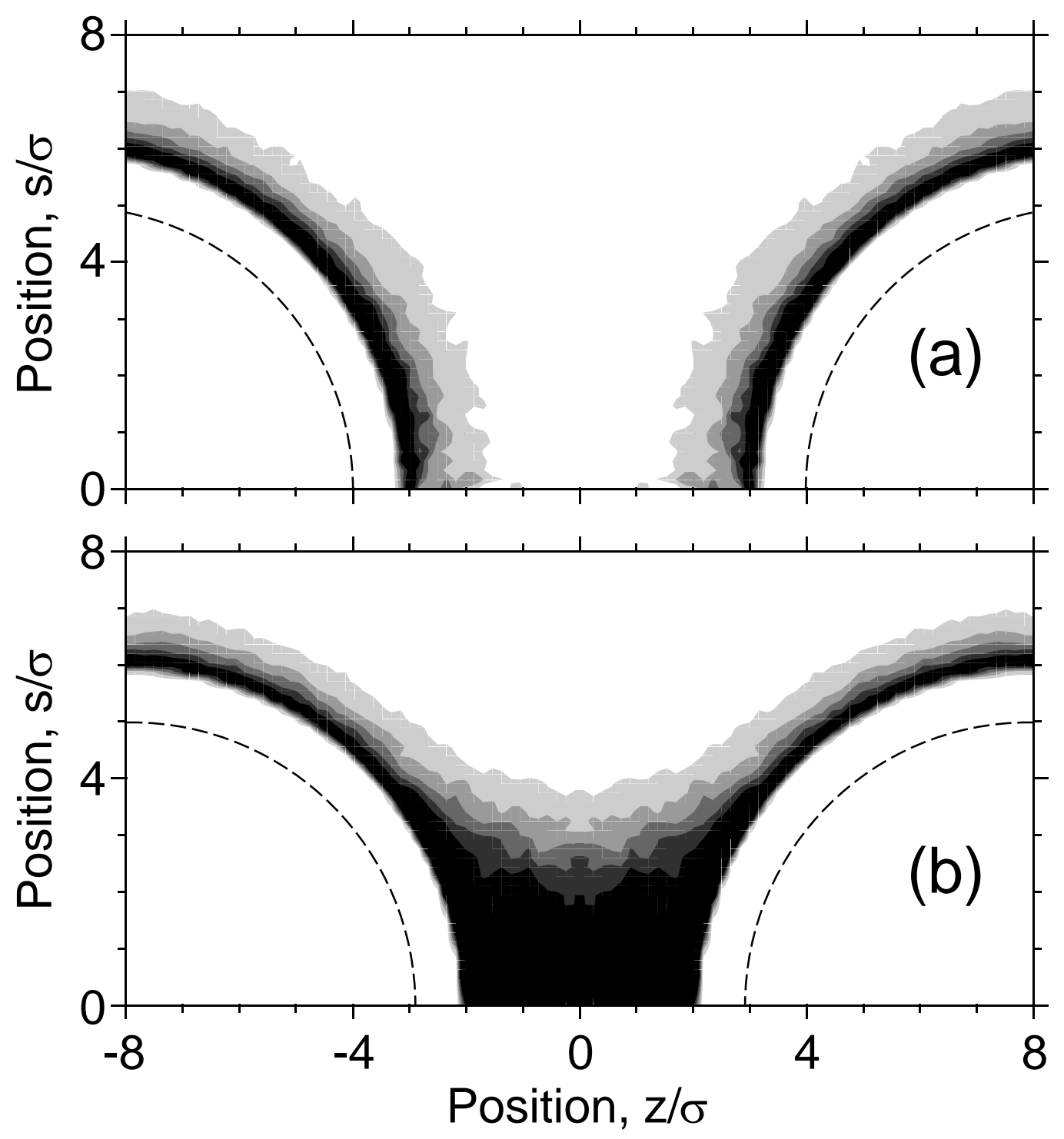
(a)

$$
\begin{aligned}
& \text { "B" rich phase } \\
& \psi=\psi_{2}
\end{aligned}
$$

"A" rich

phase

$\psi=\psi_{1}$

(b)

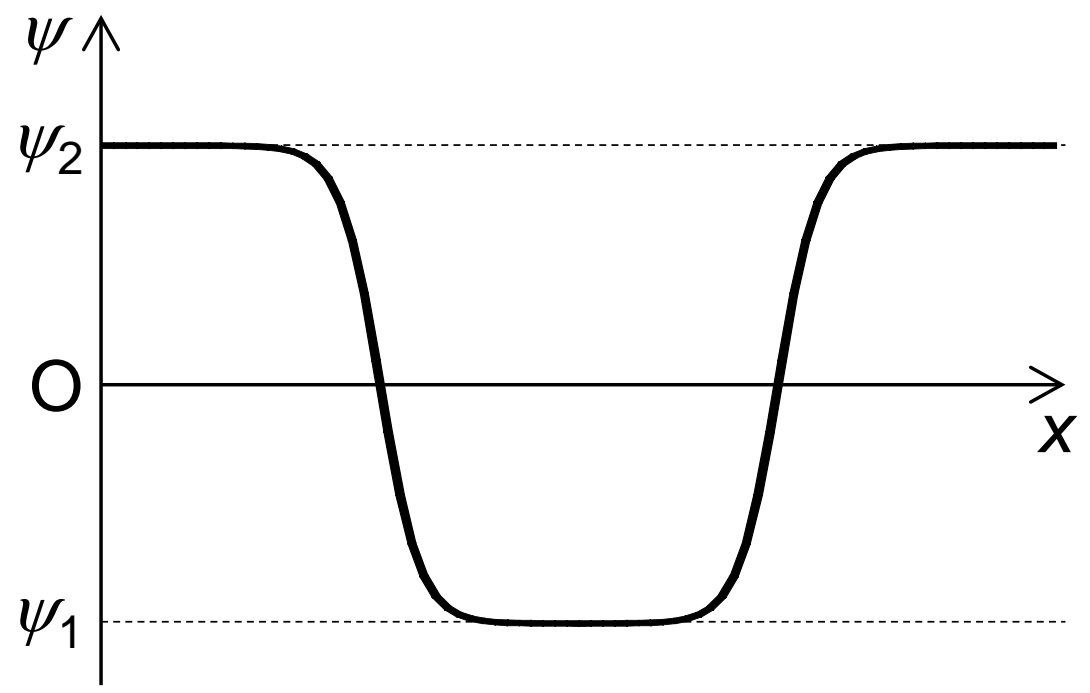

(c)

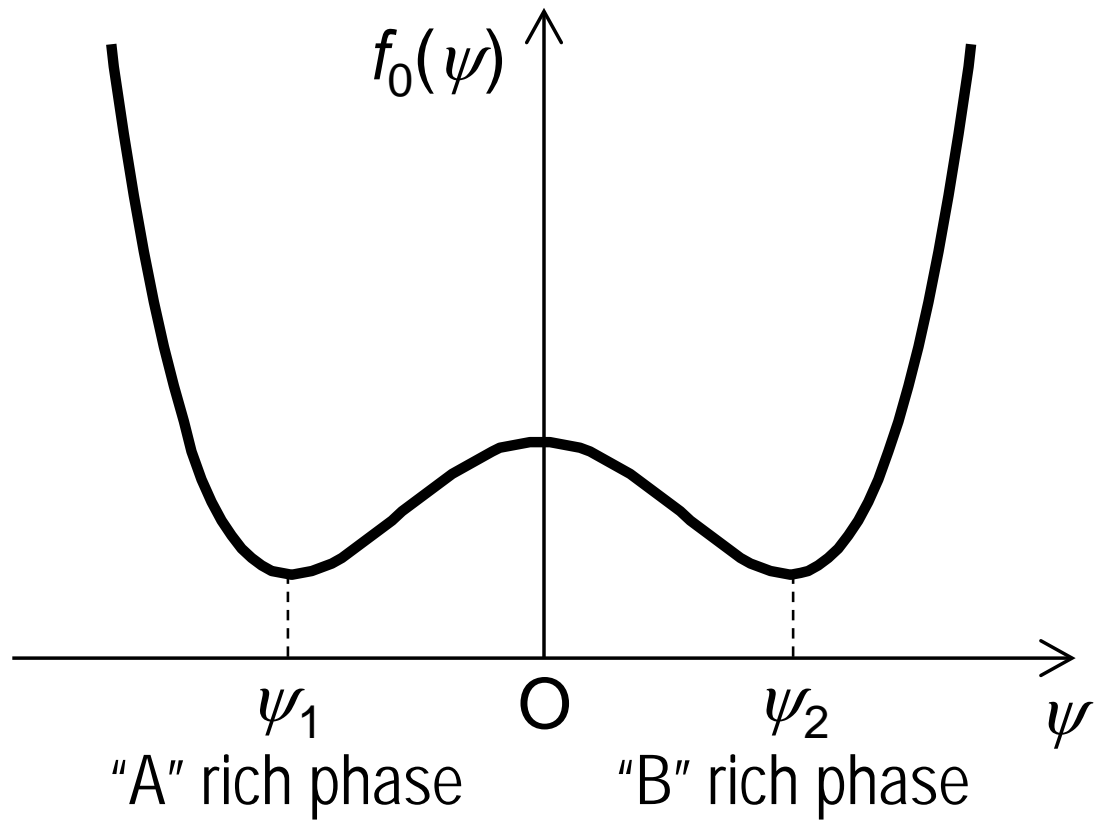


Fig. 5

(a)

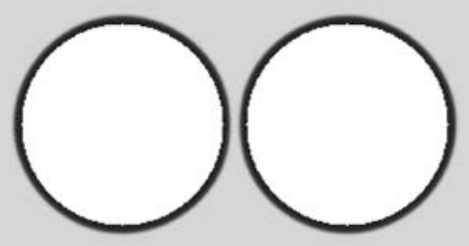

(b)

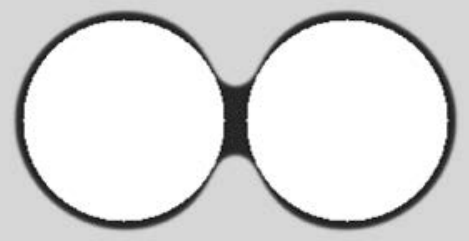


Fig. 6

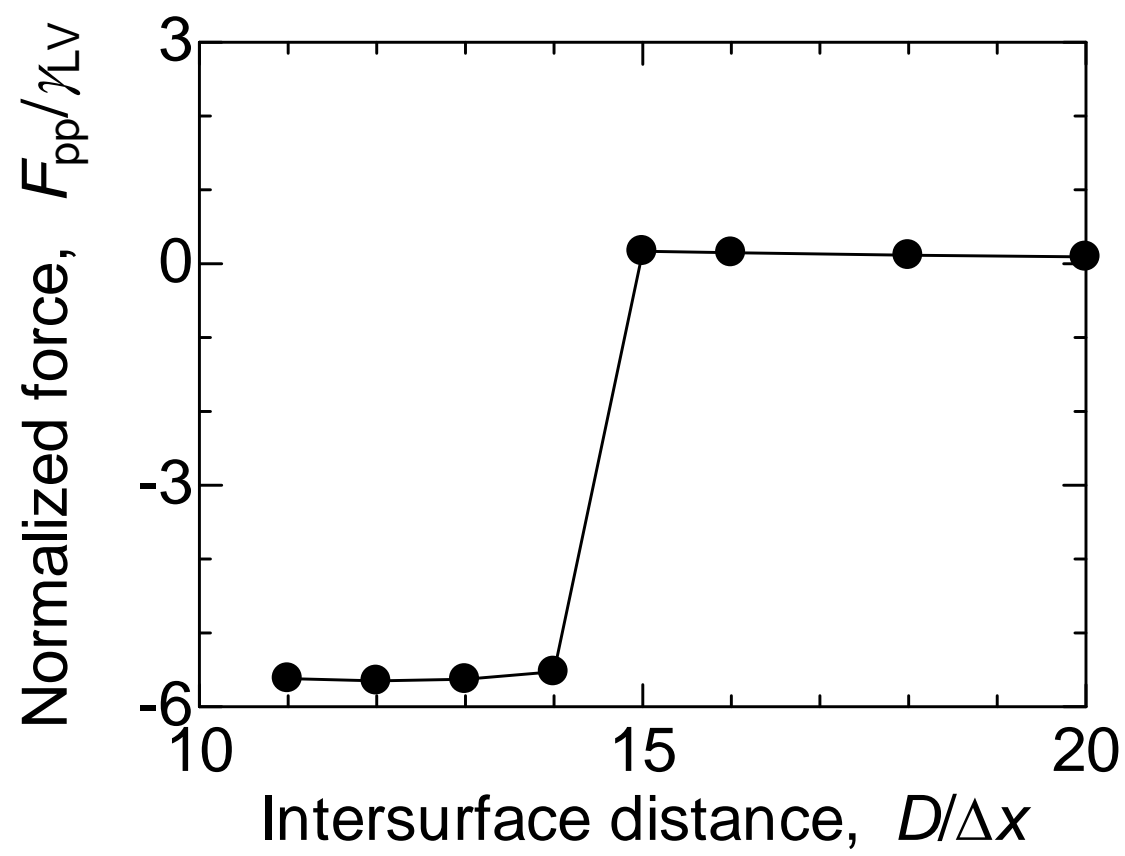


(A)

(B)

(C) 
Fig'

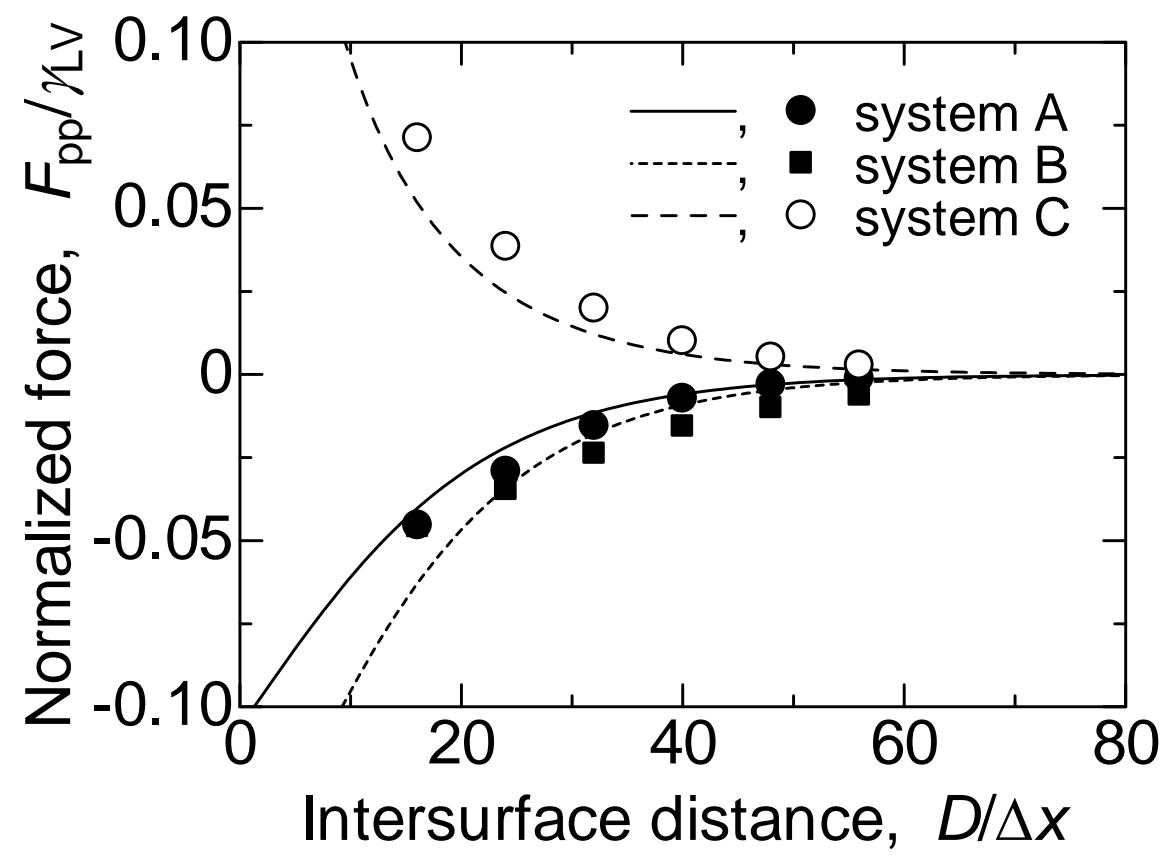




\section{(a) $t \dot{\gamma}=0$}

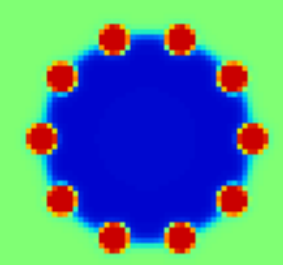

(b) $t \dot{\gamma}=3.0$

(c) $t \dot{\gamma}=4.5$

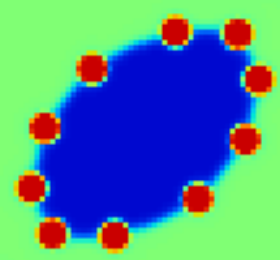

(d) $t \dot{\gamma}=6.0$

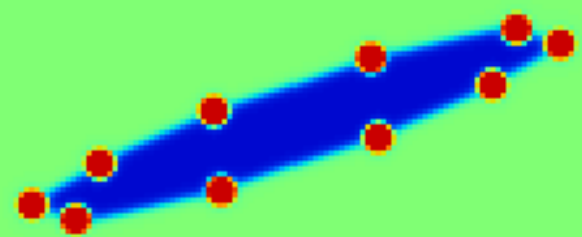

